\title{
Anidulafungin and its role in candida infections
}

REVIEW

This article was published in the following Dove Press journal:

Infection and Drug Resistance

29 June 2009

Number of times this article has been viewed

\section{Diane M Cappelletty Rose Jung}

The University of Toledo, College of Pharmacy, Toledo, OH, USA
Correspondence: Diane M Cappelletty The University of Toledo, College of Pharmacy, 280 I W. Bancroft St., MS 609, Toledo, OH 43606, USA Email diane.cappelletty@utoledo.edu

\begin{abstract}
Candida infections continue to play a significant role not only in critically ill and immunocompromised patients but also in non-compromised patients. The incidence of systemic fungal infections in the United States has been on the rise for the past 30 years. Anidulafungin and all echinocandins inhibit glucan synthase thus inhibiting the formation of 1,3- $\beta$-D-glucan which is an essential component of the fungal cell wall. The decrease in 1,3- $\beta$-D-glucan results in the osmotic lysis of the cell, resulting in fungicidal activity against candida. Anidulafungin is active against most species of candida and resistance to it is very rare. Two potential mechanisms conferring reduced susceptibility to the echinocandins are efflux and target alteration. The efflux pump associated with fluconazole resistance in Candida albicans can confer higher minimum inhibitory concentrations to caspofungin. The second mechanism of resistance is via mutations in the genes which code for 1,3 $\beta$-D-glucan synthase, specifically FKS1. Because of its spectrum of activity, fungicidal nature, and tolerability it is an attractive first-line therapeutic choice for treating candidemia in both nonneutropenic and neutropenic patients. Because it is available only parenterally its role in treating mucocutaneous candidiasis is primarily in patients unable to take oral therapy.
\end{abstract}

Keywords: anidulafungin, candida, echinocandin

Candida infections continue to play a significant role not only in critically ill and immunocompromised patients but also in non-compromised patients. The spectrum of disease caused by candida ranges from non-life-threatening mucocutaneous infections to life-threatening invasive candidiasis/candidemia. Candida remains the 4th and 5th leading cause of bloodstream infections in adult and pediatric patients, respectively. ${ }^{1,2}$ The incidence of systemic fungal infections in the United States has been on the rise the past 30 years. Between 1979 and 2000 the annual number of sepsis cases due to fungal organisms increased by $207 \% .^{3}$ In addition the number of candida-related hospitalizations increased by $52 \%$ between 2000 and $2005 .{ }^{4}$ Common risk factors for the development of systemic candidiasis or candidemia include: immunosuppression, use of broad-spectrum antibiotics, central venous catheters, TPN, disruption of mucosal membranes and extremes of age. ${ }^{5}$

The echinocandin class of anti-fungal agents (anidulafungin, caspofungin, and micafungin) was introduced into the United States and European markets in 2001. They are the most recent addition joining the azoles, polyenes, allylamines, and antimetabolites available for treating systemic fungal infections. The echinocandins are recommended as potential first-line therapy for candidemia in non-neutropenic and neutropenic patients and first- or second-line for several other candida infections. ${ }^{6}$ 
This review will focus on anidulafungin and its role in candida infections.

\section{Chemistry}

The echinocandins are synthetically modified lipopeptides which were identified from the fermentation broths of various fungi. Anidulafungin (Figure 1) is derived from Aspergillus nidulans. It is a 1-[(4R,5R)-4,5-DihydroxyN2-[[4" (pentyloxy)[1,1':4',1'-terphenyl]-4-yl]carbonyl]L-ornithine] echinocandin B. Its molecular formula is $\mathrm{C}_{58} \mathrm{H}_{73} \mathrm{~N}_{7} \mathrm{O}_{17}$, and its molecular weight is 1140.3 . $^{7}$ The echinocandins are only available parenterally and anidulafungin is available in both 50 and $100 \mathrm{mg}$ vials.

Anidulafungin is initially reconstituted with a diluent containing $20 \%$ (w/w) dehydrated alcohol in water for injection and then diluted to its final concentration of either 0.36 or $0.43 \mathrm{mg} / \mathrm{mL}$ in either $5 \%$ dextrose or normal saline. Compatibility studies with other diluents or solutions have not been performed therefore they should not be used. The maximum rate of infusion for anidulafungin is $1.1 \mathrm{mg} / \mathrm{min}$. Histamine-related adverse effects such as rash, urticaria, flushing, pruritus, dyspnea, and hypotension have been reported when the infusion rate exceeds $1.1 \mathrm{mg} / \mathrm{min}^{7}$

\section{Mechanism of action, FDA-approved indications and dosing}

Anidulafungin and all echinocandins inhibit glucan synthase thus inhibiting the formation of $1,3-\beta$-D-glucan which is an essential component of the fungal cell wall. Glucan synthase is present in fungal cells but not mammalian cells. The decrease in 1,3- $\beta$-D-glucan results in the osmotic lysis of the cell, resulting in fungicidal activity against candida. ${ }^{8}$ Anidulafungin is FDA approved for the treatment of esophageal candidiasis, candidemia, and invasive candidiasis (intra-abdominal abscess and peritonitis). ${ }^{7}$

For the treatment of esophageal candidiasis, the recommended loading dose is $100 \mathrm{mg}$ followed by the maintenance dose of $50 \mathrm{mg}$ daily. The duration of treatment should be based on the patient's clinical response with most patients being treated for $\geq 14$ days, or for $\geq 7$ days after the resolution of symptoms. For the treatment of candidemia, the recommended loading dose of $200 \mathrm{mg}$ is followed by $100 \mathrm{mg}$ daily for the duration of treatment of $\geq 14$ days after the last positive blood culture results. No dosage adjustments are needed in patients with hepatic or renal impairment regardless of severity. Anidulafungin is not dialyzed during hemodialysis. $^{7}$

\section{Spectrum of activity}

The Clinical and Laboratory Standards Institute (CLSI) established susceptibility breakpoints for the echinocandins in 2007. The breakpoint for susceptible against Candida organisms is $\leq 2 \mu \mathrm{g} / \mathrm{mL}$ for all three echinocandins and given the extremely low number of isolates with minimum inhibitory concentrations (MICs) higher than $2 \mu \mathrm{g} / \mathrm{mL}$ no breakpoints for intermediate or resistant were established. ${ }^{9}$

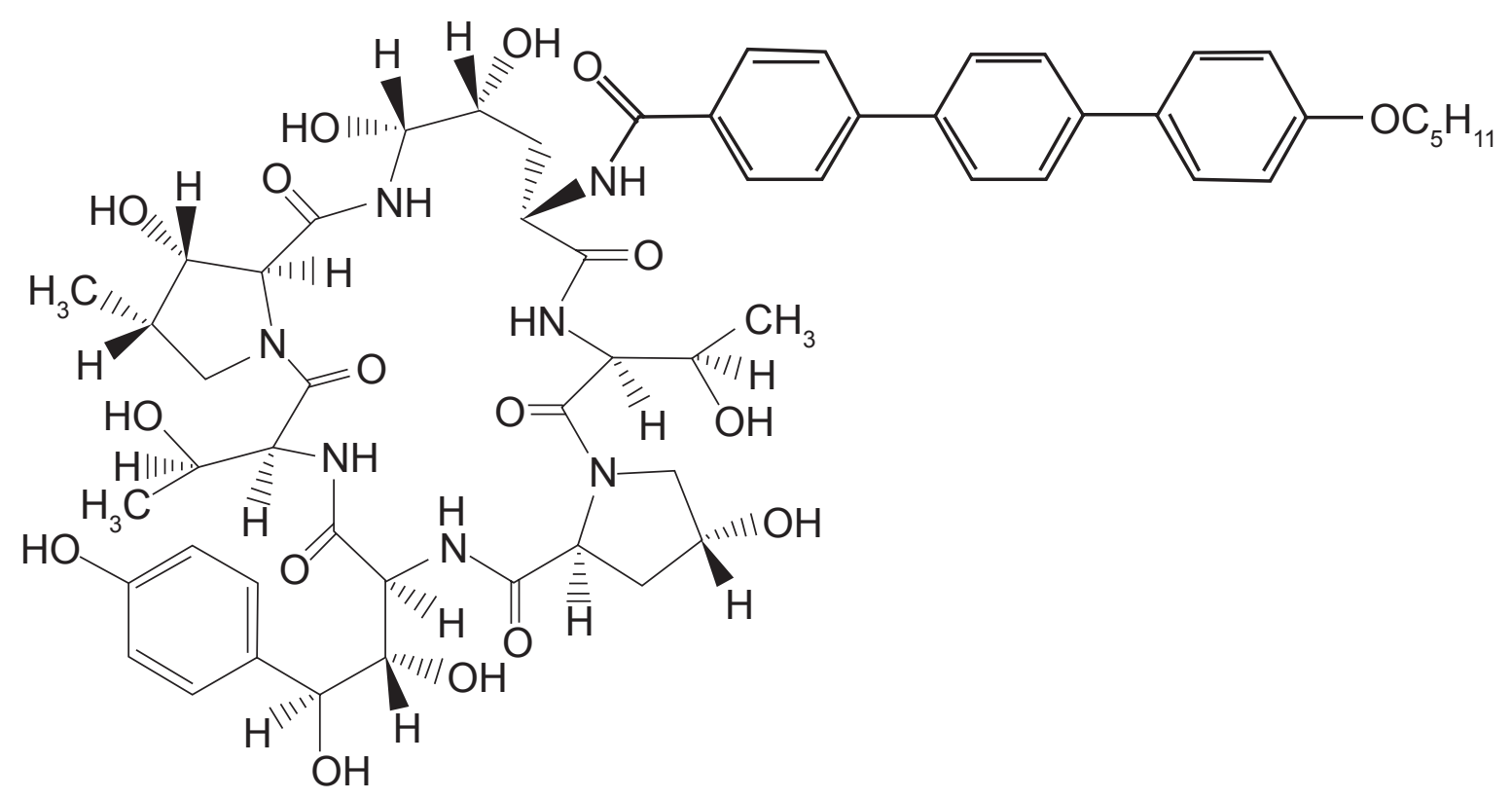

Figure I Anidulafungin chemical structure. 
Organisms with MICs $>2 \mu \mathrm{g} / \mathrm{mL}$ are considered non-susceptible. The European Committee for Antimicrobial Susceptibility Testing (EUCAST) has not established breakpoints for the echinocandins. ${ }^{10}$ The organisms to which the echinocandins are highly active include $C$. albicans, C. glabrata, C. tropicalis, C. dubliniensis, and C. krusei. In general, the $\mathrm{MIC}_{50}$ of anidulafungin against these pathogens is $\leq 0.03 \mu \mathrm{g} / \mathrm{mL}$ and the $\mathrm{MIC}_{90}$ is $\leq 0.13 \mu \mathrm{g} / \mathrm{mL} .^{11,12}$ In vitro anidulafungin is more active than caspofungin against these pathogens although this has not been clinically proven to be significant. ${ }^{11-14}$ The echinocandins are less active against $C$. parapsilosis, $C$. guilliermondii, and C. lusitaniae compared to the other Candida spp; the $\mathrm{MIC}_{50}$ and $\mathrm{MIC}_{90}$ of anidulafungin range from 0.06 to 2 and 0.25 to $2 \mu \mathrm{g} / \mathrm{mL}$, respectively. ${ }^{11-13}$ Anidulafungin is highly active against azole-resistant candida with $99 \%$ of isolates inhibited at $\leq 1 \mu \mathrm{g} / \mathrm{mL} .{ }^{11}$ CLSI recommends further testing be performed on $C$. albicans, C. tropicalis, or C. glabrata isolates in which an echinocandin MIC of 1 or $2 \mu \mathrm{g} / \mathrm{mL}$ is obtained. None of the echinocandins are active against Cryptococcus neoformans (MICs 16 to $64 \mu \mathrm{g} / \mathrm{mL}$ ) or other Cryptococcus spp. ${ }^{15}$ The echinocandins are active against Aspergillus spp. ${ }^{16-20}$ The $\mathrm{MIC}_{90}$ for anidulfungin against $A$. fumigatus is $\leq 0.25 \mu \mathrm{g} / \mathrm{mL} .{ }^{16,17}$ Activity against other species of Aspergillus is similar to that seen against fumigatus.

\section{Resistance}

Resistance to the echinocandins is rare amongst Candida spp. and identification of the mechanism(s) has resulted in contradictory information. The efflux pump associated with fluconazole resistance in C. albicans was suggested to confer higher MICs to caspofungin. ${ }^{21}$ The increase in MICs to caspofungin was minor and the isolates were still considered susceptible by CLSI breakpoint. This cross-substrate of the echinocandins to the fluconazole efflux pump did not occur with all yeast isolates expressing or hyperexpressing the efflux pump. ${ }^{22}$ The second mechanism of resistance to the echinocandins in C. albicans, C. parapsilosis and C. krusei is via mutations in the genes which code for 1,3 $\beta$-D-glucan synthase, specifically FKS1. ${ }^{23-25}$ Five candida isolates, which had MICs $>4 \mu \mathrm{g} / \mathrm{mL}$ to caspofungin were recovered from patients enrolled in a caspofungin clinical trial and all were found to have mutations in the FKS1 gene. ${ }^{23}$ Within C. parapsilosis an intrinsic mutation in FKS1 appears to be responsible for the higher MICs for the echinocandins. The mutations in the conserved hot spot 1 region of fks 1 appear to result in a glucan synthase which is less sensitive to the echinocandins and some isolates had MICs $>8 \mu \mathrm{g} / \mathrm{mL} .^{26-28}$ Another study failed to demonstrate mutations in the hot spot 1 of several isolates of $C$. parapsilosis which had higher MICs to caspofungin. ${ }^{29}$ These isolates had caspofungin and micafungin MICs of $\geq 8 \mu \mathrm{g} / \mathrm{mL}$ and the anidulafungin $\mathrm{MIC}_{90}$ for anidulafungin was $2 \mu \mathrm{g} / \mathrm{mL}$. Moudgal and colleagues also reported a $C$. parapsilosis isolate in which the caspofungin and micafungin MICs increased to $>16 \mu \mathrm{g} / \mathrm{mL}$ while anidulafungin's MIC was $2 \mathrm{mcg} / \mathrm{mL} .^{30}$ The mechanism behind this disparity in MICs of anidulafungin compared to the other 2 agents is still unknown but mutations in FKS2 and/or FKS3 may play a role.

The incidence of developing resistance during therapy is still rare and a small number of cases regarding the development of higher MICs while receiving caspofungin have been reported. The first involved $C$. parapsilosis prosthetic valve endocarditis and the patient failed caspofungin therapy. The MICs during the first hospitalization were 2, 8 and $1 \mu \mathrm{g} / \mathrm{mL}$ for caspofungin, micafungin and anidulafungin, respectively which increased to $>16 \mu \mathrm{g} / \mathrm{mL}$ for caspofungin and micafungin and $2 \mu \mathrm{g} / \mathrm{mL}$ for anidulafungin. ${ }^{30}$ Reports of failed echinocandin therapy in HIV or AIDS patients with recurrent esophagitis caused by $C$. albicans have been published. ${ }^{31-33}$ Gene sequencing in two of the cases revealed C. albicans isolates with caspofungin MICs of $\geq 8 \mu \mathrm{g} / \mathrm{mL}$ with mutation of the FKS1 gene. ${ }^{31,32}$ One of the isolates was resistant to all 3 of the echinocandins. ${ }^{32}$ Reports exist as well for other nonalbicans candida (C. glabrata, C. krusei and C. tropicalis) developing resistance during caspofungin therapy. ${ }^{34-36}$ Increases in MICs to the three echinocandins are not necessarily uniform as demonstrated by these case reports.

Potential limitations or problems with the current CLSI breakpoint of $\leq 2 \mu \mathrm{g}$ as susceptible and the methodologies recommended for susceptibility testing by both CLSI and EUCAST have been identified. Ardendrup and colleagues evaluated susceptibility methodologies on a $C$. albicans isolate from a patient who died from a fungal infection which had been treated with caspofungin. ${ }^{37}$ In addition to the EUCAST ${ }^{38,39}$ and CLSI $^{40}$ methods they evaluated Etest and agar dilution susceptibility methods. The EUCAST method resulted in a susceptible interpretation for both caspofungin and anidulafungin with MICs of $\leq 2$ and $\leq 0.125 \mu \mathrm{g} / \mathrm{mL}$, respectively. The CLSI method resulted in caspofungin and anidulafungin MICs of $\leq 2$ and $\leq 0.5 \mu \mathrm{g} / \mathrm{mL}$, respectively. Etest demonstrated MICs for both agents of $>32 \mu \mathrm{g} / \mathrm{mL}$ and agar dilution showed growth at all dilutions including $2 \mu \mathrm{g} / \mathrm{mL}$. Molecular characterization of the isolate revealed a mutation in the hot 
spot region of the FKS1 gene. ${ }^{37}$ It has been demonstrated that in the presence of serum the MICs of caspofungin increase an average of 1 - to 16-fold, micafungin 32- to 128-fold, and anidulafungin 8- to 256-fold compared to testing conditions without serum. ${ }^{41,42}$ Garcia-Effron and colleagues evaluated the susceptibility of the echinocandins in the absence and presence of serum against 14 isolates with FKS1 mutation. ${ }^{43}$ CLSI and EUCAST methodologies don't include serum in their methodologies and under these conditions $12 / 14$ isolates were susceptible to anidulafungin, $10 / 14$ to micafungin, and only $3 / 14$ to caspofungin. In the presence of serum $2 / 14$ were susceptible to anidulafungin, $1 / 14$ to micafungin, and $0 / 14$ to caspofungin. ${ }^{43}$ Therefore, current susceptibility testing methods may not detect all echinocandin non-susceptible candida isolates with the FKS1 mutation. Further evaluation is needed to determine if changing the breakpoint for micafungin and anidulafungin is warranted to detect non-susceptible candida organisms. In addition there is a need to further evaluate the role of including serum in the methodologies for susceptibility testing as well as how to interpret the data. At present, Garcia-Effron and colleagues postulate that caspofungin can be used as a surrogate marker for predicting the susceptibility of all of the echinocandins based on the premise that the echinocandins share the same target, mechanism of resistance, spectrum of activity and in vitro potency. ${ }^{43}$

\section{Pharmacodynamics}

Results from four phase 2 and 3 studies of anidulafungin in patients with esophageal or oropharyngeal candidiasis were examined to determine a pharmacokinetic-pharmacodynamic relationship. In this study, successful treatment was defined as either resolution of signs and symptoms or endoscopic response at the completion of therapy. Multiple pharmacokinetic parameters were associated with success and included the AUC at steady state (AUCss) greater than $35 \mathrm{mg} * \mathrm{~h} / \mathrm{L}$, concentration at steady state (Css) greater than $1.5 \mu \mathrm{g} / \mathrm{mL}$, and minimum concentration $\left(\mathrm{C}_{\min }\right)$ greater than $1 \mu \mathrm{g} / \mathrm{mL}$. This study did not specify which of these pharmacokinetic parameters was most closely associated with success. Anidulafungin's potent activity against Candida spp. and its favorable pharmacokinetics allow drug exposure to be in excess of these pharmacokinetic-pharmacodynamic targets with recommended doses. Anidulafungin at the approved maintenance dosage of $50 \mathrm{mg}$ per day for esophageal candidiasis produces a Css of $2.2 \mu \mathrm{g} / \mathrm{mL}$, an AUCss of $53 \mathrm{mg}{ }^{*} \mathrm{~h} / \mathrm{L}$ and a $\mathrm{C}_{\min }$ above $1 \mu \mathrm{g} / \mathrm{mL}$ throughout the dosing interval in a typical patient. ${ }^{44}$
Similar findings were also reported in animal studies. When a pharmacokinetic-pharmacodynamic relationship was evaluated in persistently neutropenic rabbit infection model with disseminated candidiasis, $100 \%$ efficacy was achieved with a $\mathrm{C}_{\max }$ of approximately $2 \mu \mathrm{g} / \mathrm{mL}$, an $\mathrm{AUC}_{0-24}$ of $8 \mu \mathrm{g} * \mathrm{~h} / \mathrm{mL}$, and a time of 12 hours with plasma concentration above the minimum fungicidal concentration (MFC) for the test organism. Again, this model was not able to discern which parameter most closely associated with optimal antifungal activity. ${ }^{45}$ Another study of pharmacodynamic characterization in a neutropenic murine model of disseminated candidiasis reported concentration-dependent efficacy against C. albicans and C. glabrata. In this study, the $\mathrm{C}_{\text {max }}$ :MIC and the $\mathrm{AUC}_{0-24}$ :MIC ratios were most strongly associated with antifungal activity. ${ }^{46} \mathrm{~A}$ post anti-fungal effect (PAFE) exists for the echinocandins and candida. Against Candida spp., the PAFE is concentration-dependent with higher concentrations resulting in longer PAFEs. ${ }^{47,48}$ At concentrations equal to or greater than the MIC of the Candida organism the PAFE was greater than 12 hours for most isolates tested. ${ }^{47,48}$

An Eagle effect is an in vitro paradoxical effect, and above a particular concentration instead of a decrease in organism, an increase occurs. This effect has been observed with the echinocandins with both yeast and filamentous fungi. ${ }^{49-51}$ Stevens and colleagues postulated that the high concentrations derepressed resistance mechanisms. ${ }^{52}$ The clinical significance of this phenomenon is unknown but appears to be negligible and further evaluation may be warranted.

\section{Pharmacokinetics}

Pharmacokinetic studies of anidulafungin have been conducted in healthy volunteers, patients with invasive fungal infection, renal or hepatically impaired patients, and in children. Results from these studies demonstrate that anidulafungin has poor and variable absorption after oral administration. However, when administered intravenously, absorption concentrations are predictable and exposure is increased linearly with dose.

\section{Pharmacokinetics in healthy volunteers}

The pharmacokinetics of $\left[{ }^{14} \mathrm{C}\right]$ anidulafungin at a mean dose $88.3 \mathrm{mg}$ (range: 87.6 to $88.7 \mathrm{mg}$ ) and $95 \mu \mathrm{g} \mathrm{Ci}$ were evaluated in 9 healthy male volunteers. Following a single intravenous dose of anidulafungin, a mean $\mathrm{C}_{\max }$ of $3.63 \mu \mathrm{g} / \mathrm{mL}$, mean AUC of $92.5 \mu \mathrm{g} * \mathrm{~h} / \mathrm{mL}$, a large volume of distribution $(\mathrm{Vd})$ of $32.6 \mathrm{~L}$ and a long-mean terminal elimination half-life $\left(t_{1 / 2}\right)$ of 27.7 hours were reported..$^{53}$

In addition to the aforementioned pharmacokinetic study in healthy volunteers, other experiments that included in vitro 
degradation, in vitro human cytochrome P450 inhibition, in vitro incubation with rat and human hepatocytes, and mass balance studies in rats were conducted to characterize anidulafungin clearance. The results revealed that anidulafungin undergoes slow chemical degradation to a primary inactive product, which is likely further degraded by plasma peptidases. The primary degradation product and subsequent ones produced by plasma peptidases are assumed to be void of antifungal activity. The products from degradation and less than $10 \%$ of the unchanged drug are eliminated into feces via biliary excretion. Although the intact drug has a $t_{1 / 2}$ of approximately 1 day, the degradation products are thought to persist in the body for a longer period of time. Anidulafungin does not undergo hepatic metabolism nor interact with cytochrome P450 isoenzymes. Renal elimination of the drug is negligible. ${ }^{53}$

\section{Pharmacokinetics in patients with invasive fungal infections}

Data from four different phase 2 and 3 clinical studies were combined to describe the pharmacokinetic characteristics of anidulafungin in patients with invasive fungal infections. A total of 225 patients received various anidulafungin regimens consisting of a loading dose of twice the daily maintenance dose $(50,75,100 \mathrm{mg})$ as treatment for esophageal candidiasis (129 patients), invasive candidiasis (87 patients), invasive aspergillosis ( 7 patients) or azole-refractory mucosal candidiasis ( 2 patients). All doses were administered intravenously at a rate of $1 \mathrm{mg} / \mathrm{min}^{.54}$

The results revealed that a two-compartment model with first-order elimination best described the disposition of anidulafungin. The estimated pharmacokinetic parameters were similar to those observed in healthy volunteers. The clearance was estimated to be $0.946 \mathrm{~L} / \mathrm{h}$, the $\mathrm{Vd}$ at steady state was $33.2 \mathrm{~L}$, and the $\mathrm{t}_{1 / 2}$ was 25.9 hours. When demography (age, sex, weight, race), concomitant drugs, and study participation were taken into consideration, the central volume of distribution increased with increasing body weight. In addition, clearance was increased in male subjects, patients with increased body weight and patients who participated in the invasive candidiasis study. Patients in the invasive candidiasis study were hospitalized, older, had higher body weight, and were more acutely ill than those who participated in the esophageal candidiasis study which may have contributed to altered clearance of the drug. However, these predictors explained less than $20 \%$ of the difference in clearance rate and the differences were deemed to have little clinical significance. ${ }^{54}$
Concomitant medications that were categorized as substrates, inducers, or inhibitors of cytochrome P450 isoenzymes, including rifampin were also evaluated in this study. None of these drugs had significant impact on anidulafungin population pharmacokinetic parameters, indicating lower potential for interactions with drugs that affect cytochrome P450 isoenzymes. ${ }^{54}$

\section{Renal and hepatic impairment}

To evaluate anidulafungin pharmacokinetics in patients with hepatic insufficiency, a single intravenous dose of $50 \mathrm{mg}$ was administered to 19 subjects ( 6 mild, 6 moderate, and 7 severe hepatic impairment patients). Pharmacokinetic parameters in patients with mild or moderate impairment were not significantly different from healthy controls. On the other hand, subjects with severe hepatic impairment showed statistically significant decreases in $\mathrm{C}_{\max }(36 \%$ decrease: mean $\pm \mathrm{SD}$ $1.8 \pm 0.8$ vs $2.9 \pm 0.7 \mu \mathrm{g} / \mathrm{mL})$ and AUC (33\% decrease $46.6 \pm 14.1$ vs $70.0 \pm 13.4 \mu \mathrm{g} * \mathrm{~g} / \mathrm{mL})$ as well as, significant increases in clearance (57\% increase: $1.16 \pm 0.34 \mathrm{vs}$ $0.74 \pm 0.15 \mathrm{~L} / \mathrm{h}$ ) and volume of distribution at steady state ( $78 \%$ increase: $50.8 \pm 17.0$ vs $28.5 \pm 6.5 \mathrm{~L}$ ). However, the half-life was similar in both groups (severe hepatic impairment vs controls: $35.2 \pm 7.1$ vs $31.2 \pm 1.5$ hours). This decrease in exposure compared with control subjects were thought to be due to ascites and edema. Unfortunately, protein binding was not evaluated in this study. The reduced $\mathrm{C}_{\max }$ and AUC in these patients may be important factors to consider in the treatment of fungemia. However, anidulafungin $50 \mathrm{mg}$ per day produces levels that exceed the $\mathrm{MIC}_{90}$ of most Candida spp. throughout the dosing period. Consequently, no dosage adjustment of anidulafungin is currently recommended for any degree of hepatic impairment. ${ }^{55}$

Anidulafungin's pharmacokinetic profile was evaluated in 21 patients with varying degrees of renal function. Patients with mild (51 to $70 \mathrm{~mL} / \mathrm{min}$ ), moderate (31 to $50 \mathrm{~mL} / \mathrm{min}$ ), severe $(\leq 30 \mathrm{~mL} / \mathrm{min})$ renal impairment or patients with end-stage renal disease were given a single $50 \mathrm{mg}$ dose of anidulafungin. In comparison to 8 healthy volunteers, pharmacokinetic profiles were similar among the groups. In addition, no measurable quantity of anidulafungin was present in dialysate. Therefore, due to minimal renal excretion and clearance by hemodialysis, no dosage adjustment of anidulafungin is needed in renal insufficiency. ${ }^{55}$

\section{Pediatric pharmacokinetics}

The pharmacokinetic profile of anidulafungin was studied in immunocompromised, hospitalized children with neutropenia. 
Children aged 2 to 17 years were given either a loading dose of $1.5 \mathrm{mg} / \mathrm{kg}$ (maximum $100 \mathrm{mg}$ ) followed by $0.75 \mathrm{mg} / \mathrm{kg}$ per day (maximum $50 \mathrm{mg}$ ) or a loading dose of $3 \mathrm{mg} / \mathrm{kg}$ (maximum $200 \mathrm{mg}$ ) followed by $1.5 \mathrm{mg} / \mathrm{kg}$ per day (maximum $100 \mathrm{mg}$ ). The mean duration of therapy was 8.7 days (range of 1 to 23 days). As with adults, steady-sate concentration was achieved after the loading dose. Similar concentration profile is reported in pediatric patients receiving doses of $0.75 \mathrm{mg} / \mathrm{kg}$ per day and adults receiving $50 \mathrm{mg}$ per day as well children receiving $1.5 \mathrm{mg} / \mathrm{kg}$ per day and adults $100 \mathrm{mg}$ per day. The half-life was approximately 20 hours which was slightly less than those estimated in adults, but still supports once daily dosing. Body weight affected clearance and volume of distribution. Therefore, for children aged 2 years and older, anidulafungin should be dosed based on body weight and no dosage adjustment is recommended based on age. ${ }^{56}$

\section{Clinical trials}

\section{Esophagitis}

One randomized, double-blind, non-inferiority trial comparing anidulafungin to fluconazole therapy was assessed for esophageal candidiasis. ${ }^{57}$ Anidulafungin $100 \mathrm{mg}$ loading dose followed by $50 \mathrm{mg}$ once daily ( $\mathrm{n}=249$ evaluable patients) and was compared to fluconazole $200 \mathrm{mg}$ loading dose followed by $100 \mathrm{mg}$ once daily ( $\mathrm{n}=255$ evaluable patients). The endoscopic success rates at the end of therapy (EOT) for anidulafungin and fluconazole were $97.2 \%$ and $98.8 \%$, respectively. The clinical success rates were $98.8 \%$ for anidulafungin $99.6 \%$ for fluconazole. The endoscopic exam at the 2-week follow-up of 462 patients revealed a success rate of $64.4 \%$ for anidulafungin compared to $89.5 \%$ for fluconazole which was statistically significant. ${ }^{57}$

A phase 2 open-label trial of anidulafungin for the treatment of azole-refractory mucosal candidiasis was performed. ${ }^{58}$ Nineteen patients were enrolled and received anidulafungin $100 \mathrm{mg}$ loading dose followed by $50 \mathrm{mg}$ once daily. Seventeen of 18 patients (94\%) of patients with oropharyngeal candidiasis and $11 / 12$ patients $(92 \%)$ with esophageal candidiasis achieved clinical success at the end of therapy. The clinical success at the 10- to 14-day follow-up was $8 / 18(44 \%)$ with oropharyngeal candidiasis and $6 / 12$ patients $(50 \%)$ with esophageal candidiasis. ${ }^{58}$

\section{Candidemia/invasive candidiasis}

Two studies evaluating the efficacy of anidulafungin for candidemia or invasive candidiasis have been performed. The first was a randomized, dose ranging study in adult patients with doses of $50 \mathrm{mg}, 75 \mathrm{mg}$, or $100 \mathrm{mg}$ once daily of anidulafungin. ${ }^{59}$ In the modified-intent-to-treat (MITT) analysis there were 37,40 , and 39 patients in the $50 \mathrm{mg}$, $75 \mathrm{mg}$, and $100 \mathrm{mg}$ dosing groups, respectively. A loading dose of twice the maintenance dose was administered on day 1 in each dosage group. Candidemia was the most prevalent infection occurring in $94 \%$ of patients, $10 \%$ (12 patients) had positive tissue cultures, $4 \%$ (5 patients) had both positive tissue and blood cultures, and 1 patient had a prosthetic hip infection. C. albicans accounted for $53 \%$ of the infections followed by C. glabrata (31\%), C. tropicalis (9\%), C. parapsilosis (9\%), C. krusei (4\%), then others at $3 \%$. Global response was defined as both clinical and microbiological success and was assessed at EOT and follow-up. At EOT the global response for the 83 evaluable patients was $84 \%, 90 \%$, and $89 \%$ with the $50 \mathrm{mg}, 75 \mathrm{mg}$, and $100 \mathrm{mg}$ doses, respectively. At follow-up, the global response of the 68 evaluable patients decreased to $72 \%, 85 \%$, and $83 \%$ with the $50 \mathrm{mg}, 75 \mathrm{mg}$, and $100 \mathrm{mg}$ doses, respectively. ${ }^{59}$

The second study was a randomized, prospective, noninferiority study comparing anidulafungin to fluconazole for candidemia or invasive candidiasis. ${ }^{60}$ Patients aged 16 to 91 years received either $200 \mathrm{mg}$ on day 1 followed by $100 \mathrm{mg}$ once daily of anidulafungin or $800 \mathrm{mg}$ on day 1 followed by $400 \mathrm{mg}$ once daily of fluconazole for at least 14 days from improvement of symptoms and negative cultures. The primary outcome was a successful global response which was defined as both clinical success (resolution of signs and symptoms of invasive candidiasis and no need for additional systemic antifungal therapy) and microbiologic success (eradication of candida species present at baseline which was determined on follow-up culture or the presumed eradication if cultures were not available) at the end of intravenous therapy. Secondary outcomes were global response at the end of all therapy, 2 and 6 weeks follow-up. In the MITT analysis 127 patients received anidulafungin and 118 received fluconazole. Candidemia was the most prevalent infection occurring in 116/127 (91.3\%) of patients receiving anidulafungin and 103/118 (87\%) of patients receiving fluconazole. Seven $(6 \%)$ and 11 patients $(9 \%)$ had candida recovered from other sterile body fluids or sites in the anidulafungin and fluconazole groups, respectively. Three percent in both groups had candida in both the blood and a sterile site. C. albicans was the predominant pathogen in patients receiving either anidulafungin $(64 \%)$ or fluconazole $(59 \%)$. The other pathogens in the anidulafungin arm were C. glabrata $(16 \%)$, C. tropicalis $(12 \%)$, C. parapsilosis $(10 \%)$. The pathogens in the fluconazole arm were slightly different in frequency 
with C. glabrata (25\%), C. tropicalis (9\%), C. parapsilosis (14\%), however this was not statistically different. A successful global response at the end of intravenous therapy was $75.6 \%$ (96/127 patients) in the anidulafungin arm and $60.2 \%$ (71/118 patients) in the fluconazole arm which was statistically significant. Anidulafungin demonstrated higher successful global response than fluconazole at each of the secondary assessments: EOT (74.0\% vs $56.8 \%)$, 2-week follow-up (64.6\% vs 49.2\%), and 6-week follow-up (55.9\% vs $44.1 \%$ ) although at 6 weeks the difference was not statistically significant. Mortality was higher in the patients receiving fluconazole $(37 / 118,31.4 \%)$ than anidulafungin $(29 / 127,22.8 \%)$ however this was not significant. In addition, more patients died in the first 10 days in the fluconazole arm (14) compared to 5 in the anidulafungin group. This study demonstrated anidulafungin was more efficacious than fluconazole at end of intravenous therapy for treating candidemia/invasive candidiasis. A curiosity of the study is the clinical response rates for C. albicans and C. glabrata. In the anidulafungin arm the success rates were $81.1 \%$ and $56.3 \%$ for albicans and glabrata, and in the fluconazole arm were $62.3 \%$ and $50 \%$, respectively. It appears the primary difference in global response was due to the poor response in fluconazole treated patients infected with C. albicans. One study site enrolled 25 patients which accounted for $10 \%$ of the MITT population. Fifteen patients received anidulafungin and 14 had a successful global response and only 5 of the 10 patients who received fluconazole had a successful global response. Statistical analysis did not reveal a study site bias. However, if those 25 patients are removed from the analysis then there is no difference in global response between the two therapies. According to FDA guidelines, a second study demonstrating this exceptional outcome of anidulafungin over fluconazole would be required in order to prove superiority.

\section{Invasive candidiasis/candidemia in neutropenic patients}

In a neutropenic mouse model of invasive candidiasis, anidulafungin demonstrated good activity against 3 strains of C. glabrata; 1 was resistant to fluconazole and 1 was resistant to amphotericin B. ${ }^{61}$ Clinical trials with anidulafungin enrolled so few patients with neutropenia that no assessment could be made therefore anidulafungin does not have a FDA indication for treating candidemia or invasive candidiasis in neutropenic patients. Despite this lack of indication the Infectious Diseases Society of America has recommended anidulafungin as a potential first-line therapy for the treatment of candidemia in neutropenic patients. ${ }^{6}$ However, anidulafungin is not indicated in the guidelines for the empiric treatment of suspected invasive candidiasis in neutropenic patients, nor is it recommended for prophylaxis for solid-organ transplant recipients, patients hospitalized in intensive care units, neutropenic patients receiving chemotherapy, and stem cell transplant recipients at risk of candidiasis. ${ }^{6}$

\section{Drug interactions}

Anidulafungin is neither a substrate nor an inhibitor of the cytochrome P450 enzyme system or of P-glycoprotein. Therefore, it is unlikely that anidulafungin will alter the pharmacokinetics of drugs that influence cytochrome P450 isoenzymes or be affected by them. Several studies evaluated the influence of anidulafungin on the metabolism of rifampin, cyclosporine, tacrolimus liposomal amphotericin B and voriconazole. These investigations do not report any significant alterations in the pharmacokinetics of either the tested agent or of anidulafungin.

\section{Rifampin}

In the aforementioned population pharmacokinetic study, concomitant medications taken by 225 patients were categorized as substrates, inducers, or inhibitors of cytochrome P450 and evaluated for their effect on clearance of anidulafungin. Rifampin is a potent inducer and therefore, was evaluated separately. A total of 27 patients $(12 \%)$ were taking rifampin during the study. Anidulafungin clearance was not affected by concomitant treatment with substrates, inhibitors, or inducers of the cytochrome P450 isoenzymes, including rifampin. ${ }^{54}$

\section{Cyclosporine}

The interaction of anidulafungin and cyclosporine was evaluated in 12 healthy volunteers. Subjects were given anidulafungin $200 \mathrm{mg}$ on day 1 then $100 \mathrm{mg}$ once daily intravenously on days 2 to 8 and cyclosporine $1.25 \mathrm{mg} / \mathrm{kg}$ orally twice daily on days 5 to 8 . One subject was withdrawn from the study on day 6 due to slight increases in hepatic transaminase levels. After concomitant administration of cyclosporine, the mean AUC of anidulafungin was $22 \%$ higher, the mean $\mathrm{C}_{\min }$ was $43 \%$ higher, the clearance was $16 \%$ lower. These alterations in anidulafungin pharmacokinetics were not considered clinically significant and subsequently, no dosage adjustments were recommended. The effect of anidulafungin on cyclosporine pharmacokinetics was not evaluated in this study. ${ }^{62}$ 


\section{Tacrolimus}

The potential interaction between anidulafungin and tacrolimus was evaluated in 36 healthy male volunteers. Subjects received tacrolimus $5 \mathrm{mg}$ orally on days 1 and 13 and anidulafungin $200 \mathrm{mg}$ on day 4 followed by $100 \mathrm{mg}$ once daily intravenously on days 5 to 13 . There were no significant differences in any of the pharmacokinetic parameters measured with or without co-administration of tacrolimus and anidulafungin. Therefore, no dosage adjustment is recommended..$^{63}$

\section{Liposomal amphotericin B}

The effect of co-administration of anidulafungin and liposomal amphotericin B was evaluated in 17 patients with invasive aspergillosis. Anidulafungin (100 mg once daily) and liposomal amphotericin B ( $5 \mathrm{mg} / \mathrm{kg}$ per day) were administered concurrently until resolution of signs or symptoms of aspergillosis or for a total of 90 days. Co-administration of these two antifungal agents was well tolerated by all subjects. ${ }^{64}$

\section{Voriconazole}

A combination of anidulafungin and voriconazole was evaluated in 17 healthy male volunteers. In a blinded, randomized, crossover design, subjects received anidulafungin with placebo, voriconazole with placebo, and anidulafungin with voriconazole. Voriconazole was administered orally $400 \mathrm{mg}$ every 12 hours on day 1 followed by $200 \mathrm{mg}$ every 12 hours on days 2 to 4 . Anidulafungin was given intravenously $200 \mathrm{mg}$ on day 1 followed by $100 \mathrm{mg}$ per day on days 2 to 4 . There were no significant differences in pharmacokinetic parameters when subjects received anidulafungin alone or in combination with voriconazole or voriconazole alone or in combination with anidulafungin. Co-administration of anidulafungin and voriconazole was well tolerated. ${ }^{65}$

\section{Safety}

Anidulafungin is well tolerated with few adverse effects. Abnormal liver function tests and hypokalemia are the most commonly reported adverse effects at $1.5 \%$ to $5 \%$ and $3 \%$ to $10 \%$, respectively. Nausea, vomiting, and diarrhea have also been reported in $1 \%$ to $3 \%$ of patients. ${ }^{57,59,60}$ Histamine-related adverse effects such as rash, urticaria, flushing, pruritus, dyspnea, and hypotension have been reported when the infusion rate exceeds $1.1 \mathrm{mg} / \mathrm{min}^{7}$

\section{Summary}

Anidulafungin is active against most species of candida and resistance to it is very rare. Because of its spectrum of activity, fungicidal nature, and tolerability it is an attractive first-line therapeutic choice for treating candidemia in both non-neutropenic and neutropenic patients. Because it is available only parenterally its role in treating mucocutaneous candidiasis is primarily in patients unable to take oral therapy. Further studies are needed to define the role of anidulafungin in the empiric treatment of suspected invasive candidiasis in neutropenic patients, or other immunocompromised patients, candida osteomyelitis, meningitis, and endocarditis.

\section{Disclosures}

The authors declare no conflicts of interest.

\section{References}

1. Wisplinghoff $\mathrm{H}$, Bischoff T, Tallent SM, Seifert H, Wenzel RP, Edmond MB. Nosocomial bloodstream infections in US hospitals: analysis of 24,179 cases from a prospective nationwide surveillance study. Clin Infect Dis. 2004;39:309-317.

2. Smith MJ. Catheter-related bloodstream infections in children. Amer J Infect Contr. 2008;36:S173.e171-S173.e173.

3. Martin GS, Mannino DM, Eaton S, Moss M. The epidemiology of sepsis in the United States from 1979 through 2000. N Engl J Med. 2003;348:1546-1554.

4. Zilberberg MD, Shorr AF, Kollef MH. Secular trends in candidemiarelated hospitalization in the United States, 2000-2005. Infect Control Hosp Epidemiol. 2008;29:978-980.

5. Richardson MD. Changing patterns and trends in systemic fungal infections. J Antimicrob Chemother. 2005;56:i5-11.

6. Pappas PG, Kauffman CA, Andes D, et al. Clinical practice guidelines for the management of candidiasis: 2009 update by the Infectious Diseases Society of America. Clin Infect Dis. 2009;48:503.

7. Pfizer I. Eraxis (anidulafungin) package insert. New York, NY 2007.

8. Bartizal K, Gill CJ, Abruzzo GK, et al. In vitro preclinical evaluation studies with the echinocandin antifungal MK-0991 (L-743,872). Antimicrob Agents Chemother. 1997;41:2326-2332.

9. Clinical and Laboratory Standards Institute (CLSI). Reference method for broth dilution antifungal susceptibility testing of yeasts: Approved standard. 3rd. ed. Wayne, PA, 2008.

10. Subcommittee on Antifungal Susceptibility Testing (AFST) of the ESCMID European Committee for Antimicrobial Susceptibility Testing (EUCAST). EUCAST Technical Note on the method for the determination of broth dilution minimum inhibitory concentrations of antifungal agents for conidia-forming moulds. Clin Microbiol Infect. 2008;14:982-984

11. Pfaller MA, Boyken L, Hollis RJ, Messer SA, Tendolkar S, Diekema DJ. In vitro activities of anidulafungin against more than 2,500 clinical isolates of Candida spp., including 315 isolates resistant to fluconazole. J Clin Microbiol. 2005;43:5425-5427.

12. Ostrosky-Zeichner L, Rex JH, Pappas PG, et al. Antifungal Susceptibility Survey of 2,000 Bloodstream Candida Isolates in the United States. Antimicrob Agents Chemother. 2003;47:3149-3154.

13. Pfaller MA, Diekema DJ, Messer SA, Hollis RJ, Jones RN. In vitro activities of caspofungin compared with those of fluconazole and itraconazole against 3,959 clinical isolates of Candida spp., including 157 fluconazole-resistant isolates. Antimicrob Agents Chemother. 2003;47:1068-1071.

14. Cuenca-Estrella M, Rodriguez D, Almirante B, et al. In vitro susceptibilities of bloodstream isolates of Candida species to six antifungal agents: results from a population-based active surveillance programme, Barcelona, Spain, 2002-2003. J Antimicrob Chemother. 2005;55:194-199. 
15. Maligie MA, Selitrennikoff CP. Cryptococcus neoformans resistance to echinocandins: $(1,3)$ beta-glucan synthase activity is sensitive to echinocandins. Antimicrob Agents Chemother. 2005;49:2851-2856.

16. Pfaller MA, Marco F, Messer SA, Jones RN. In vitro activity of two echinocandin derivatives, LY303366 and MK-0991 (L-743,792), against clinical isolates of Aspergillus, Fusarium, Rhizopus, and other filamentous fungi. Diagn Microbiol Infect Dis. 1998;30:251-255.

17. Serrano MdC, Valverde-Conde A, Chavez MM, et al. In vitro activity of voriconazole, itraconazole, caspofungin, anidulafungin (VER002, LY303366) and amphotericin B against aspergillus spp. Diagn Microbiol Infect Dis. 2003;45:131-135.

18. Diekema DJ, Messer SA, Hollis RJ, Jones RN, Pfaller MA. Activities of caspofungin, itraconazole, posaconazole, ravuconazole, voriconazole, and amphotericin B against 448 recent clinical isolates of filamentous fungi. J Clin Microbiol. 2003;41:3623-3626.

19. Arikan S, Yurdakul P, Hascelik G. Comparison of two methods and three end points in determination of in vitro activity of micafungin against Aspergillus spp. Antimicrob Agents Chemother. 2003;47: 2640-2643.

20. Odabasi Z, Paetznick VL, Rodriguez JR, Chen E, Ostrosky-Zeichner L. In vitro activity of anidulafungin against selected clinically important mold isolates. Antimicrob Agents Chemother. 2004;48:1912-1915.

21. Schuetzer-Muehlbauer M, Willinger B, Krapf G, Enzinger S, Presterl E, Kuchler K. The Candida albicans Cdr2p ATP-binding cassette (ABC) transporter confers resistance to caspofungin. Molecul Microbiol. 2003;48:225-235.

22. Niimi K, Maki K, Ikeda F, et al. Overexpression of Candida albicans CDR1, CDR2, or MDR1 does not produce significant changes in echinocandin susceptibility. Antimicrob Agents Chemother. 2006;50: 1148-1155.

23. Park S, Kelly R, Kahn JN, et al. Specific substitutions in the echinocandin target Fks1p account for reduced susceptibility of rare laboratory and clinical Candida spp. isolates. Antimicrob Agents Chemother 2005;49:3264-3273.

24. Douglas CM, D'Ippolito JA, Shei GJ, et al. Identification of the FKS1 gene of Candida albicans as the essential target of 1,3-betaD-glucan synthase inhibitors. Antimicrob Agents Chemother 1997;41: 2471-2479.

25. Balashov S, V, Park S, Perlin DS. Assessing resistance to the echinocandin antifungal drug caspofungin in Candida albicans by profiling mutations in FKS1. Antimicrob Agents Chemother. 2006;50:2058-2063.

26. Garcia-Effron G, Katiyar SK, Park S, Edlind TD, Perlin DS. A Naturally Occurring Proline-to-Alanine Amino Acid Change in Fks1p in Candida parapsilosis, Candida orthopsilosis, and Candida metapsilosis Accounts for Reduced Echinocandin Susceptibility. Antimicrob Agents Chemother. 2008;52:2305-2312.

27. Perlin DS. Resistance to echinocandin-class antifungal drugs. Drug Resistance Updates. 2007;10:121.

28. Kahn JN, Garcia-Effron G, Hsu MJ, Park S, Marr KA, Perlin DS. Acquired echinocandin resistance in a Candida krusei isolate due to modification of glucan synthase. Antimicrob Agents Chemother. 2007;51:1876.

29. Ghannoum MA, Chen A, Buhari M, et al. Differential in vitro activity of anidulafungin, caspofungin and micafungin against Candida parapsilosis isolates recovered from a burn unit. Clin Microbiol Iinfect. 2009; $15: 274$.

30. Moudgal V, Little T, Boikov D, Vazquez JA. Multiechinocandin- and Multiazole-Resistant Candida parapsilosis Isolates Serially Obtained during Therapy for Prosthetic Valve Endocarditis. Antimicrob Agents Chemother. 2005;49:767-769.

31. Miller CD, Lomaestro BW, Park S, Perlin DS. Progressive esophagitis caused by Candida albicans with reduced susceptibility to caspofungin. Pharmacother. 2006;26:877-880.

32. Laverdiere M, Lalonde RG, Baril JG, Sheppard DC, Park S, Perlin DS. Progressive loss of echinocandin activity following prolonged use for treatment of Candida albicans oesophagitis. J Antimicrob Chemother. 2006;57:705-708.
33. Hernandez S, Lopez-Ribot JL, Najvar LK, McCarthy DI, Bocanegra R, Graybill JR. Caspofungin resistance in Candida albicans: correlating clinical outcome with laboratory susceptibility testing of three isogenic isolates serially obtained from a patient with progressive Candida esophagitis. Antimicrob Agents Chemother. 2004;48: 1382-1383.

34. Krogh-Madsen M, Arendrup MC, Heslet L, Knudsen JD. Amphotericin B and caspofungin resistance in Candida glabrata isolates recovered from a critically ill patient. Clin Infect Dis. 2006;42:938-944.

35. Hakki M, Staab JF, Marr KA. Emergence of a Candida krusei isolate with reduced susceptibility to caspofungin during therapy. Antimicrob Agents Chemother. 2006;50:2522-2524.

36. Pasquale T, Tomada JR, Ghannoun M, Dipersio J, Bonilla H. Emergence of Candida tropicalis resistant to caspofungin. J Antimicrob Chemother. 2008;61:219.

37. Arendrup MC, Garcia-Effron G, Buzina W, et al. Breakthrough Aspergillus fumigatus and Candida albicans double infection during caspofungin treatment: laboratory characteristics and implication for susceptibility testing. Antimicrob Agents Chemother. 2009; $53: 1185$.

38. Cuenca-Estrella M, Moore CB, Barchiesi F, et al. Multicenter evaluation of the reproducibility of the proposed antifungal susceptibility testing method for fermentative yeasts of the Antifungal Susceptibility Testing Subcommittee of the European Committee on Antimicrobial Susceptibility Testing (AFST-EUCAST). Clin Microbiol Iinfect. 2003;9:467.

39. Cuenca-Estrella M, Arendrup MC, Chryssanthou E, et al. Multicentre determination of quality control strains and quality control ranges for antifungal susceptibility testing of yeasts and filamentous fungi using the methods of the Antifungal Susceptibility Testing Subcommittee of the European Committee on Antimicrobial Susceptibility Testing (AFST-EUCAST). Clin Microbiol Iinfect. 2007;13:1018.

40. National Committee for Clinical Laboratory Standards. Reference method for broth dilution antifungal susceptibility testing of yeasts. Approved standard M27-A2, 2nd ed. National Committee for Clinical Laboratory Standards, Wayne, PA; 2002.

41. Paderu P, Garcia-Effron G, Balashov S, Delmas G, Park S, Perlin DS Serum Differentially Alters the Antifungal Properties of Echinocandin Drugs. Antimicrob Agents Chemother. 2007;51:2253-2256.

42. Odabasi Z, Paetznick V, Rex JH, Ostrosky-Zeichner L. Effects of serum on in vitro susceptibility testing of echinocandins. Antimicrob Agents Chemother. 2007;51:4214-4216.

43. Garcia-Effron G, Park S, Perlin DS. Correlating echinocandin MIC and kinetic inhibition of fks1 mutant glucan synthases for Candida albicans: implications for interpretive breakpoints. Antimicrob Agents Chemother. 2009;53:112-122.

44. Dowell JA, Stogniew M, Krause DS, Henkel T. Anidulafungin (ANID) pharmacokinetic (PK)/Pharmacodynamic (PD) correlation: Treatment of esophageal candidiasis (EC). 43rd Interscience Conference on Antimicrob Agents Chemother. Washington, DC: American Society of Microbiology; 2003:A1578.

45. Groll AH, Mickiene D, Petraitiene R, et al. Pharmacokinetic and pharmacodynamic modeling of anidulafungin (LY303366): reappraisal of its efficacy in neutropenic animal models of opportunistic mycoses using optimal plasma sampling. Antimicrob Agents Chemother. 2001;45:2845-2855.

46. Andes D, Diekema DJ, Pfaller MAet al. In vivo pharmacodynamic characterization of anidulafungin in a neutropenic murine candidiasis model. Antimicrob Agents Chemother 2008;52:539-550.

47. Ernst EJ, Roling EE, Petzold CR, Keele DJ, Klepser ME. In vitro activity of micafungin (FK-463) against Candida spp: microdilution, time-kill, and postantifungal-effect Studies. Antimicrob Agents Chemother. 2002;46:3846-3853.

48. Ernst EJ, Klepser ME, Pfaller MA. Postantifungal effects of echinocandin, azole, and polyene antifungal agents against Candida albicans and Cryptococcus neoformans. Antimicrob Agents Chemother. 2000;44:1108-1111. 
49. Hall GS, Myles C, Pratt K, J, Washington JA. Cilofungin (LY121019), an antifungal agent with specific activity against Candida albicans and Candida tropicalis. Antimicrob Agents Chemother. 1988;32: 1331-1335.

50. Ramage G, VandeWalle K, Bachmann SP, Wickes BL, LopezRibot JL. In vitro pharmacodynamic properties of three antifungal agents against preformed Candida albicans biofilms determined by time-kill studies. Antimicrob Agents Chemother. 2002;46: 3634-3636.

51. Wiederhold NP, Kontoyiannis DP, Chi J, Prince RA, Tam VH, Lewis RE. Pharmacodynamics of caspofungin in a murine model of invasive pulmonary aspergillosis: evidence of concentration-dependent activity. J Infect Dis. 2004;190:1464-1471.

52. Stevens DA, Espiritu M, Parmar R. Paradoxical effect of caspofungin: reduced activity against Candida albicans at high drug concentrations. Antimicrob Agents Chemother. 2004;48:3407-3411.

53. Damle BD, Dowell JA, Walsky RL, Weber GL, Stogniew M, Inskeep PB. In vitro and in vivo studies to characterize the clearance mechanism and potential cytochrome P450 interactions of anidulafungin. Antimicrob Agents Chemother. 2009;53:1149.

54. Dowell JA, Knebel W, Ludden T, Stogniew M, Krause D, Henkel T. Population pharmacokinetic analysis of anidulafungin, an echinocandin antifungal. J Clin Pharmacol. 2004;44:590-598.

55. Dowell JA, Stogniew M, Krause D, Damle B. Anidulafungin does not require dosage adjustment in subjects with varying degrees of hepatic or renal impairment. J Clin Pharmacol. 2007;47:461-470.

56. Benjamin DK, Jr, Driscoll T, Seibel NL, et al. Safety and pharmacokinetics of intravenous anidulafungin in children with neutropenia at high risk for invasive fungal infections. Antimicrob Agents Chemother. 2006;50:632-638
57. Krause DS, Simjee AE, van Rensburg C, et al. A randomized, double-blind trial of anidulafungin versus fluconazole for the treatment of esophageal candidiasis. Clin Infect Dis. 2004;39:770.

58. Vazquez JA, Schranz JA, Clark K, Goldstein BP, Reboli A, Fichtenbaum C. A phase 2, open-label study of the safety and efficacy of intravenous anidulafungin as a treatment for azole-refractory mucosal candidiasis. J Acquir Immune Defic Syndr. 2008;48:304-309.

59. Krause DS, Reinhardt J, Vazquez JA, et al. Phase 2, randomized, dose-ranging study evaluating the safety and efficacy of anidulafungin in invasive candidiasis and candidemia. Antimicrob Agents Chemother. 2004:48:2021-2024.

60. Reboli AC, Rotstein C, Pappas PG, et al. Anidulafungin versus fluconazole for invasive candidiasis. $N$ Engl J Med. 2007;356:2472-2482.

61. Gumbo T, Drusano GL, Liu W, et al. Anidulafungin pharmacokinetics and microbial response in neutropenic mice with disseminated candidiasis. Antimicrob Agents Chemother. 2006;50:3695.

62. Dowell JA, Stogniew M, Krause D, Henkel T, Weston IE. Assessment of the safety and pharmacokinetics of anidulafungin when administered with cyclosporine. J Clin Pharmacol. 2005;45:227-233.

63. Dowell JA, Stogniew M, Krause D, Henkel T, Damle B. Lack of pharmacokinetic interaction between anidulafungin and tacrolimus. J Clin Pharmacol. 2007;47:305-314.

64. Herbrecht R, Graham D, Schuster M. Safety and tolerability of combination anidulafungin (ANID) and liposomal amphotericin B (LAmB) for the treatment of invasive aspergillosis (IA). Tandem Bone Marrow Transplantation Meetings. Orlando, FL: American Society for Blood and Marrow Transplantation, 2004:91.

65. Dowell JA, Schranz J, Baruch A, Foster G. Safety and pharmacokinetics of coadministered voriconazole and anidulafungin. J Clin Pharmacol. 2005;45:1373-1382.
Infection and Drug Resistance

\section{Publish your work in this journal}

Infection and Drug Resistance is an international, peer-reviewed openaccess journal that focuses on the optimal treatment of infection (bacterial, fungal and viral) and the development and institution of preventive strategies to minimize the development and spread of resistance. The journal is specifically concerned with the epidemiology of antibiotic

\section{Dovepress}

resistance and the mechanisms of resistance development and diffusion in both hospitals and the community. The manuscript management system is completely online and includes a very quick and fair peer-review system, which is all easy to use. Visit http://www.dovepress.com/ testimonials.php to read real quotes from published authors. 